\title{
A Service Oriented Method for Health Care Network Governance
}

\author{
Hannes Schlieter ${ }^{1}$, Stephan Bögel ${ }^{2}$, and Werner Esswein ${ }^{1}$ \\ ${ }^{1}$ TU Dresden, Münchener Platz, Schumann-Bau, 01062 Dresden, Germany \\ Tel.: +49 (0)351 463-32173, +49 351 463-37671 \\ \{Hannes.Schlieter, Werner.Esswein\} atu-dresden.de \\ ${ }^{2}$ Virtimo AG, Schlesische Str. 29-30, 10997 Berlin, Germany \\ Tel.: +49 (0) 3060922556 \\ Boegelevirtimo.de
}

\begin{abstract}
Governance and compliance of health care networks gain more and more attention in the IS research. The configuration of medical care workflow systems and the compliance check of care processes according to national and international guidelines is the motivation for this paper. We are following a process model based approach for the management of health care networks. We present a service-based method for the compliance check of process models and enable a configuration of information systems with process models. The application of the method as well as the discussion of the practical benefits is illustrated by a real world case.
\end{abstract}

Keywords: Conceptual Models, Vertical Integration, Conflict Management, Health Care Network, Service-oriented method, Care process compliance.

\section{Introduction}

\subsection{Situation}

A major problem in the health care sector is to improve the medical care and at the same time reduce costs. To improve the medical care Clinical Practice Guidelines (CPGs) and Clinical Pathways (CPs) are used to communicate best practices and to establish a standard care quality. CPGs provide decision guidance for health care provider that is based on evident practice for specific indications (diseases). CPs describe clinical processes for a specific diagnosis that is adapted to the local situation, e.g. in a specific hospital. CPGs are typically released by national or international medical associations like the European Stroke Association. CPs are created by the medical care provider according to their local processes.

\subsection{Conceptual Models in the Health-Care Sector}

Conceptual modeling is used in CPGs and CPs to standardize and communicate clinical processes (Schlieter \& Esswein 2010; Wollersheim et al. 2005). A conceptual modeling language is the combination of a technical language with a modeling 
grammar, and results in the creation of conceptual models. Hence, the conceptual model comprises formal-structural representation and non-formal content (Frank 1999). Conceptual models are an accepted instrument to describe organizations in its various aspects such as structures and behavior.

In health care domain different models are constructed simultaneously for same indication by different groups of interests. These models are complementary and in relation but are not totally equal. We call this set of models "model landscape" and their implicit relation "hidden relation". The integration of CPGs and CPs is demanded in the literature but has not been completely realized (Wollersheim et al. 2005). Process compliance in clinical context becomes more and more important in a competitive health care market. Firstly, compliant $\mathrm{CP}$ can be used as an instrument to implement evident medical knowledge in organization. Secondly, they build a basis for continuous process improvement and measurement. CPGs could thus be used to govern the right implementation of CPs in practice. The CPGs are the regulatory element for the governance of clinical processes. To the extent of our knowledge there are no holistic approaches that provide the alignment or integration between CPG and CP models.

To illustrate the context of the present situation in a "big picture", we want to introduce the life cycle of the medical care processes (see figure 1). The life cycle can be divided into three phases or layers. Starting point is the model landscape in the first phase where models are isolated and inconsistent. With the help of the proposed method, the models are vertically integrated. The integration on the model layer is the prerequisite for the next two phases. On the organizational layer in phase 2 the result of the model integration is used to guide the compliance implementation. To be compliant with the guidelines operational procedures and organizational structures have to be adjusted. On the technical layer the hospital information systems is configured using the integrated models to support the execution of the medical care processes.

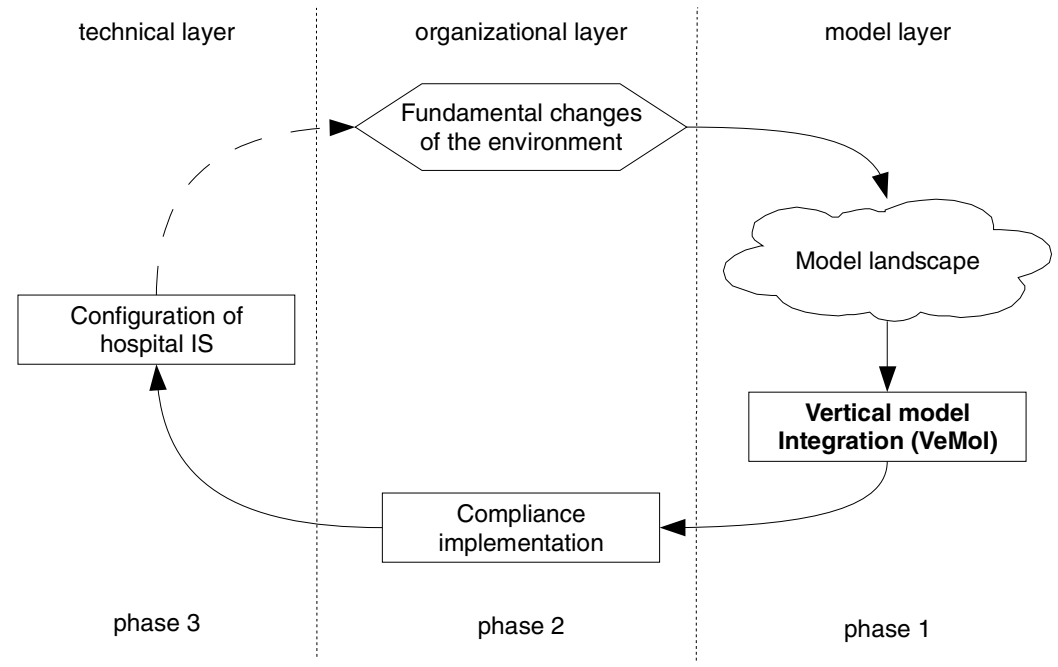

Fig. 1. The Life Cycle of Medical Care Processes 
The life cycle starts, if the environment fundamentally changes, e.g. due to new laws. Because of the exceptional relevance of the model layer for the organizational and technical layer this research in progress paper focuses on the first step, i.e. the integration of these models. In this paper we aim to present the research goal and a proposal for a way to achieve the vertical model integration. Our research should build the linking element between those studies which demand a consideration of the CPGs while CPs development (Lelgemann \& Ollenschläger 2006; Schnabel et al. 2003) and are dealing with discussion to transfer the CPG content in the formal machine-readable - syntax (Seyfang \& Miksch 2007; Peleg et al. 2003).

\subsection{Research Method and Outline}

The research can be assigned to the field of design science (Hevner et al. 2004; Peffers et al. 2007). Knowledge is gained hereby the creation and evaluation of artifacts in the form of languages, models, methods or systems. The investigation aims for the usefulness of the constructing artifact in the sense of a problem solution. We use a case scenario to make the requirements analysis and evaluate our artifact. In a descriptive case study (Yin 2003) we examine the situation of Stroke Network Saxony (SOS-NET).

In the first part of the paper, the theoretical foundations of integration conflicts are discussed shortly. Afterwards we examine the situation of the case scenario and derive requirements for a vertical model integration method. The paper finishes with the proposal for a vertical model integration method (VeMoI) and an outlook on further research.

\section{Theoretical Foundations}

\subsection{Vertical vs. Horizontal Model Integration}

In the context of model integration vertical and horizontal integration of model layers needs to be distinguished (Bögel and Esswein 2010). We speak of vertical integration if the integrated model layers concern the same part of the universe of discourse, but differ in the viewpoint. If we look on CPGs, the universe of discourse is a specific disease, while the viewpoint is the evident guideline viewpoint on this specific disease. For a corresponding CP, the universe of discourse is the same disease, but the viewpoint is that of a specific process in a certain hospital.

In contrast, the horizontal integration of two models on the same layer, differ in the universe of discourse but have the same viewpoint. Heterogeneity in vertical integration is therefore a result of different viewpoints.

\subsection{Integration Conflicts}

The heterogeneity of models leads to integration conflicts between those models. Diverse classifications of integration conflicts can be found in the literature. A common classification distinguishes type, structural and name conflicts (Pfeiffer and Gehlert 2005; Pfeiffer and Becker 2008; Rosemann 2002; Hars 1994). If a part of the real world is modeled semantically different in two models this is denominated as struc- 
tural or semantic conflict (Pfeiffer and Gehlert 2005). These can further be distinguished as dependency conflicts, abstraction conflicts and level of detail (Kashyap and Sheth 1996).

Dijkman (2008) considers the horizontal integration of similar process models. He differentiates authorization, activity and control-flow conflicts. Weidlich et al. (2009) extend the list by process and data conflicts. Those conflicts relate to constructs of a modeling language. Others like (Pfeiffer and Becker 2008) are differentiating homonym, abstraction, separation, type, synonym, annotation and control-flow conflicts.

Table 1. Overview of Integration Conflicts

\begin{tabular}{|l|l|l|l|}
\hline & Domain language & Modeling Language & Solutions \\
\hline Syntax & $\begin{array}{l}\text { Name conflict } \\
\text { (homonym } \\
\text { conflict, synonym } \\
\text { conflict) }\end{array}$ & Type conflict & $\begin{array}{l}\text { Ontologies, } \\
\text { syntactical model } \\
\text { comparison }\end{array}$ \\
\hline Semantics & - & $\begin{array}{l}\text { Authorization conflict, } \\
\text { Activity conflict, Process } \\
\text { conflict, } \\
\text { Data conflict, Dependency } \\
\text { conflict, Abstraction } \\
\text { conflict, Level of detail } \\
\text { conflict, Annotation conflict, } \\
\text { Control-flow conflict, Order } \\
\text { conflict, Separation conflict }\end{array}$ & VeMoI \\
\hline Pragmatics & - & - & - \\
\hline
\end{tabular}

We consolidate these classifications according to table 1 . The previously mentioned conflicts relate either to symbols of the domain languages or to concepts of modeling languages.

In the dimension of semiotics, the conflicts can be classified by looking at their occurrence. Name and type conflicts can be identified at the level of the syntax (for example, through the comparison of strings or two type identifiers). All the other conflicts mentioned (Dijkman 2008; Pfeiffer and Becker 2008) can only be detected by looking at the semantic layer. No conflicts could be found on the pragmatic layer. The mapping between syntax and semantics is an on-going process executed by human beings. Humans use symbols corresponding to his or her intentions and therefore constitute semantics (Holenstein 1982; Wittgenstein 1922). As a consequence, for the solution of these conflicts, the next semiotic level has to be considered.

\section{Case Study}

In industrialized countries, the stroke is the third major cause of death and the most frequent reason of lasting physical handicaps (Kolominsky-Rabas et al. 2006). In acute stroke care, the capabilities of therapy worsen with each minute the stroke is not 
diagnosed and treated accordingly. An immediate medical intervention is necessary to avoid consequential damage.

This is especially important for radiological findings. Further characteristics for an acute stroke are short delay for decision-making, limited local transfer possibility and symptoms that are difficulty diagnosed. In 2007, the SOS-NET was founded to build a telemedical infrastructure for regional stroke care. The goal of the network is to treat all stroke patients consitently and independently from distance to next stroke expert over the whole region. Within two years the SOS-NET is growing to an important stroke care network in Saxony (Germany).

Presently, there are fourteen hospitals participating in the network. 580 telemedical consultations were carried out in 2009. The suspicion of stroke was confirmed in $79 \%$ of the telemedical presented patients. 89 lyses-therapies were indicated. If the indication can be done at early stage, this therapy allows an almost complete rehabilitation of special stroke cases.

The different suspension of employees and the differences of the technical infrastructure necessitate a defined responsibilities and process within the network. The stroke center as the core of network allocates CPGs for the partners of the network (stroke units) to assure a network-wide high standard clinical care.

In figure 2 , the situation is depicted in which the models interrelate. The framework shows three different and initially independent model layers. On the topmost layer professional associations and local experts create (CPG). CPs are located on the middle layer and are used by the hospital staff. The hospital information system (HIS) is shown on the bottom layer. Typically, on the technical layer, the HIS could be configured by workflow models via a XML-Transformation. The care process life cycle (see figure 1) is referenced through the different phases. In phase 1 the clinical pathways are checked against the appropriate CPG. The compliant models are than used to implement the organizational structures and in phase 3 to configure the hospital information system.

The integration of the layers delivers great potential to improve the recommendations themselves and thus the quality of clinical care. The following cases reflect the main goals of integration in the SOS-NET:

Case 1: Compliance check: The vertical integration makes a check of models concerning superordinate layer possible. The models are compliant, if all necessary advises are considered in the underling layer. Compliance should guarantee that the goals of care are reached as it is defined in the subordinate layer. Compliance is no structural or syntactical conformity. It depends largely on the content wise correspondence.

Case 2: Context sensitive help: If a link between the different layers exists, user can track where information come from. They can also get further information for special steps of the treatment or clinical decisions.

Case 3: Support of business-process-reengineering (BPR): In BPR it is necessary to know the current situation of the processes and the limits of BPR. The integration model could help to determine the flexibility for the restructure. On the other, the integration could help to identify weak parts in the processes. 


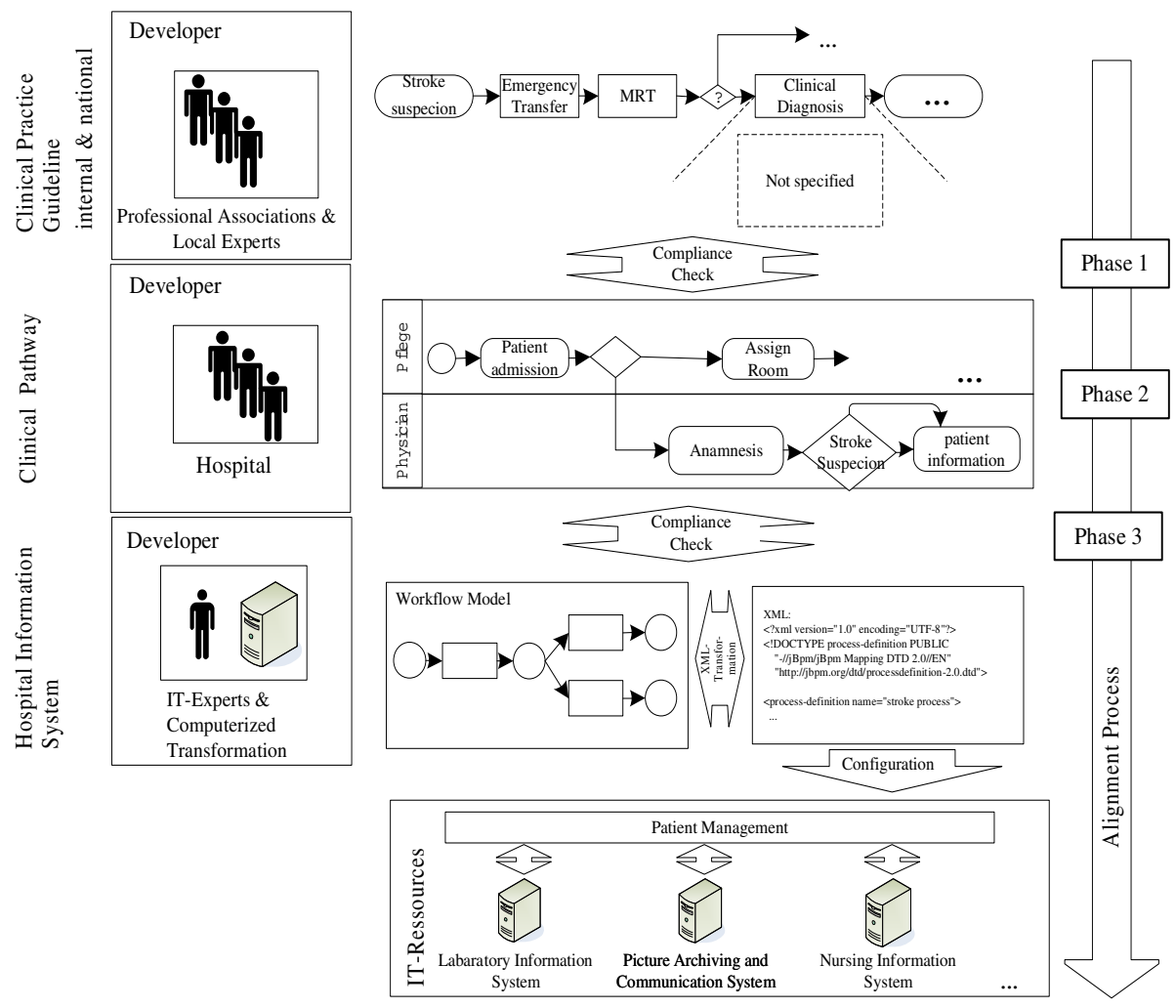

Fig. 2. Framework for medical care processes

\section{Requirement Analysis}

In this section, we want to present the results of our requirements analysis (figure 3 ). The analysis was done with the $i^{*}$ - (i-star)-framework. It provides a method to structure relations of actors and their influence to goals, to describe decompositions as well as means-ends-relation between tasks and resources which are necessary to achieve the (soft)goal(s) (Yu 2009). The starting point of the analysis is the goal to treat patient with the "Best Clinical Care". From this main goal, the goal of the evidencebased utilization of CP depends. This means integration between CPGs and CPs is needed.

Kaveh et al. showed that CPG are permanent under revision (Kaveh et al. 2007). Since CP guide evident clinical care, they need to be up-to-date against the CPG which was supported by the integration. Further the integration would encourage a context sensitive help in business-workflow as well as business-process-reengineering projects. To achieve these goals, a method would help to reconstruct the inherent relationships between the models. This can only be done with the knowledge about the conflicts ("conflict identification"). To allow a successful application of the method, a procedure model should document the main steps of the method. 
Supporting techniques and heuristics are necessary for the automated and nonautomated parts of the method. The case study shows that various modeling languages are used to describe clinical recommendations. Thus the method should be applied to various modeling languages.

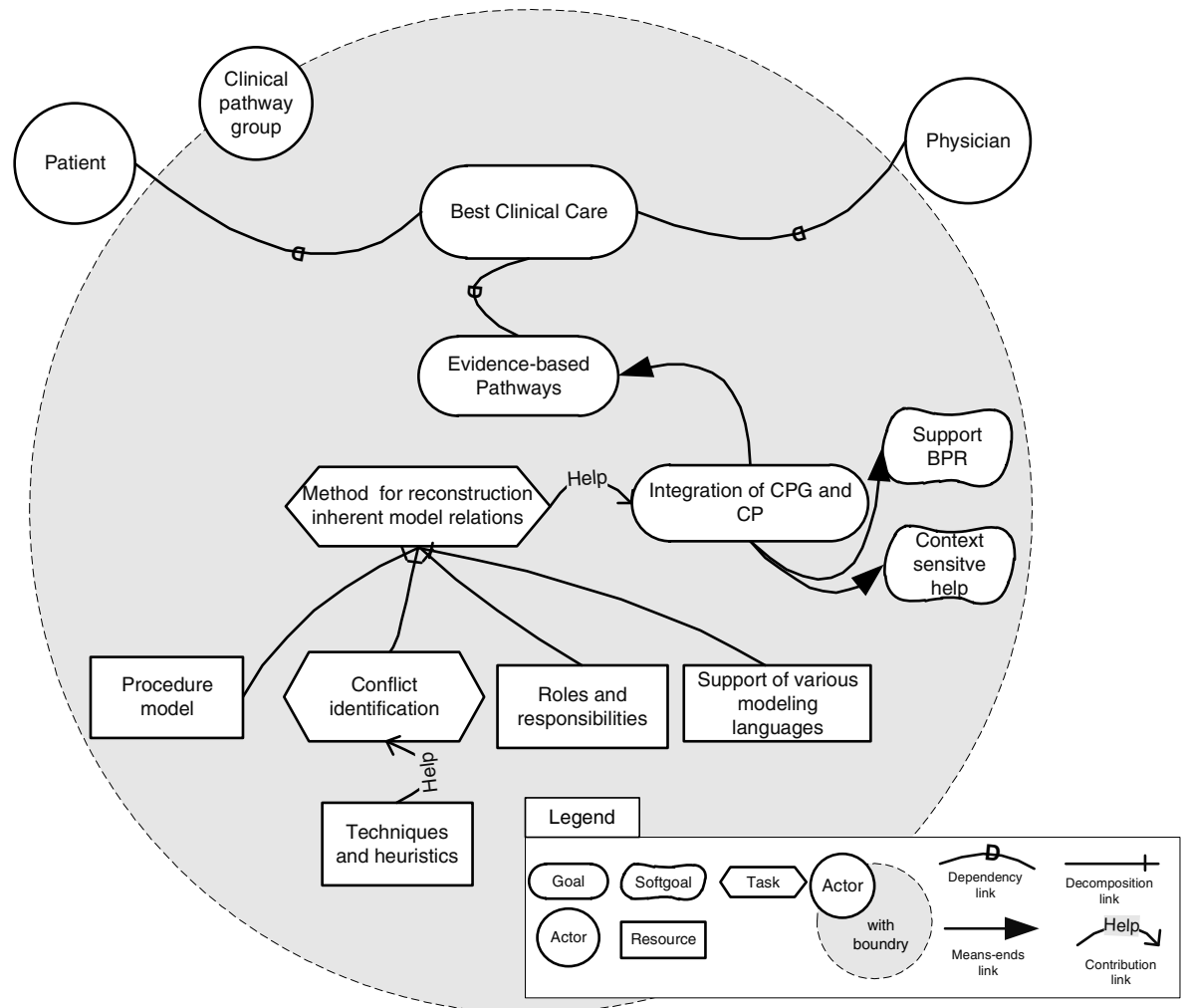

Fig. 3. Summary of the requirements $(i *$-model)

\section{Intermediary Results - VEMOI}

We want to introduce a service-oriented method based on the following considerations. First the health care network requires a distributed architecture. Second we gain flexibility in the orchestration of the services so we can integrate preliminary work, e.g. the integration of ontologies for the resolving of name conflicts. We can also change non-automated steps with automated steps, if such a service is available. A hospital or care network may also decide to outsource certain services like the classification step. In summary, our procedure model is described by orchestrating services and not by modeling a process diagram. Following this approach a method fragment is represented by a service. In Figure 4 three services: "classification", "anchor 
identification" and "domain conflict identification" and their orchestration are depicted. These step lead to the reconstruction of the inherent model relations. The central element of our approach "VeMol" is the anchor model, which allows to represent inter-model relations as well as possible conflict situations. It guides also the participators of integration project to find and to eliminate conflict situations.

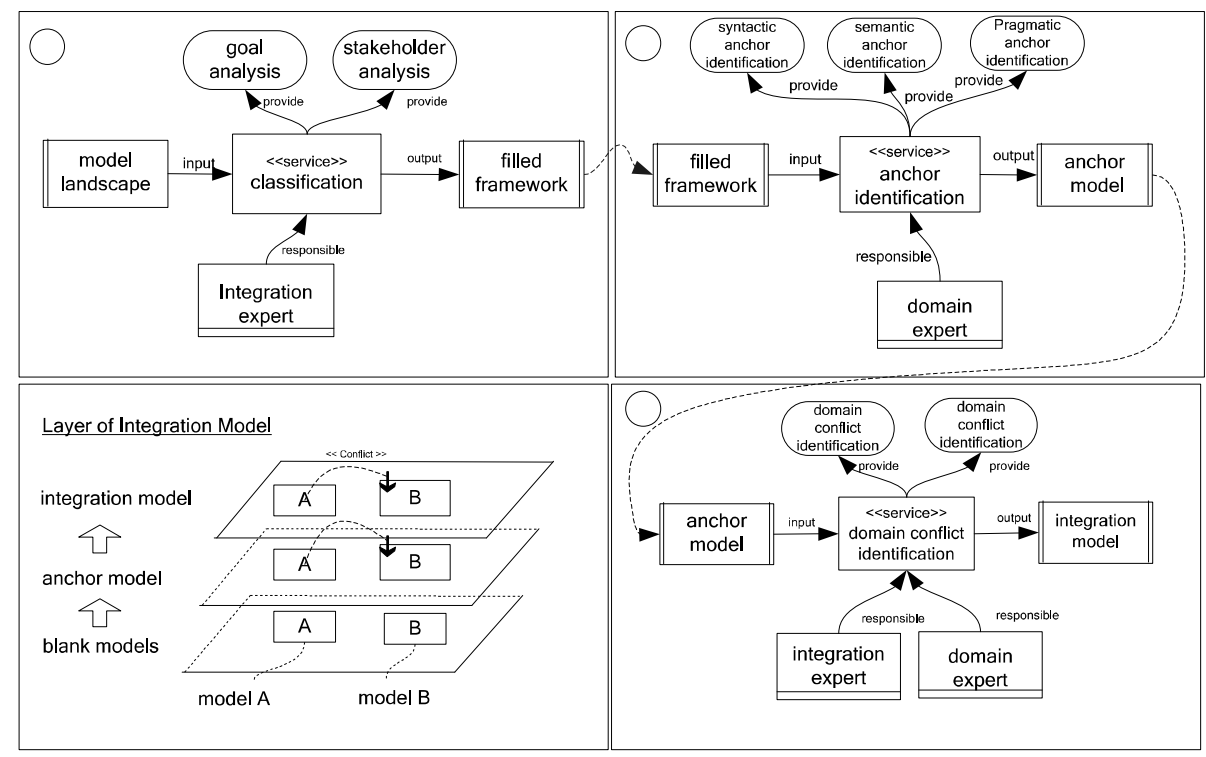

Fig. 4. The VeMoI - Service Model

\section{Expected Contribution and Outlook}

Expected contribution of this work is the proposal of the VeMoI method for the vertical integration of medical care process models. This is an important prerequisite for the organizational compliance implementation and the model-based configuration of the hospital information system. With this method we want to design a domain language based approach for conflict handling in heterogeneous model landscapes.

The identified domain conflicts should allow compliance checks against the CPG. The process of identifying conflicts also should shows relations between two models that hopefully can be used for context sensitive enrichment of the medical care process models. As a third use case the relations and domain conflicts might be used for business process reengineering. The enriched process models probably allow the identification of reengineering potential.

Next steps our research are the finalization of the integration method and its evaluation in the context of a greater case study inside the stroke and a cancer network. 


\section{References}

Batini, C., Lenzerini, M., Navathe, S.B.: A comparative analysis of methodologies for database schema integration. ACM Computing Surveys (CSUR) 18(4), 364 (1986)

Beimborn, D., et al.: Die Bedeutung des Alignment von IT und Fachressourcen in Finanzprozessen Eine empirische Untersuchung. Wirtschaftsinformatik 48(5), 331-339 (2006)

Pfeiffer, D., Becker, J.: Solving the conflicts of distributed process modelling - towards an integrated approach. In: Proceedings of the 16th European Conference on Information Systems (ECIS 2008), Galway, Ireland (2008)

Dijkman, R.: Diagnosing differences between business process models. In: Dumas, M., Reichert, M., Shan, M.-C. (eds.) BPM 2008. LNCS, vol. 5240, pp. 261-277. Springer, Heidelberg (2008)

Frank, U.: Conceptual modelling as the core of the information systems discipline-perspectives and epistemological challenges. In: Proceedings of the Fifth Americas Conference on Information Systems (AMCIS 1999), pp. 13-15 (August 1999)

Hars, A.: Referenzdatenmodelle. Gabler, Wiesbaden (1994)

Hevner, A.R., et al.: Design science in information systems research. Management Information Systems Quarterly 28(1), 75-106 (2004)

Holenstein, E.: On the cognitive underpinnings of language. Semiotica 41(1-4), 107-134 (1982)

Kashyap, V., Sheth, A.: Semantic and schematic similarities between database objects: a context-based approach. The VLDB Journal 5(4), 276-304 (1996)

Katona, G.: Psychologie der Relationserfassung und des Vergleichens, Johann Ambrosius Barth (1924)

Kaveh, D., et al.: How quickly do systematic reviews go out of date? A Survival Analysis. Anal. of Internal Medicine 147(4), 224-233 (2007)

Kolominsky-Rabas, P.L., et al.: Lifetime Cost of Ischemic Stroke in Germany: Results and National Projections From a Population-Based Stroke Registry: The Erlangen Stroke Project. Stroke 37(5), 1179-1183 (2006)

Lelgemann, M., Ollenschläger, G.: Evidenzbasierte Leitlinien und Behandlungspfade. Der Internist 47(7), 690-698 (2006)

Mertens, P.: Die zwischenbetriebliche Kooperation und Integration bei der automatisierten Datenverarbeitung. Hain, Meisenheim am Glan (1966)

Peffers, K., et al.: A Design Science Research Methodology for Information Systems Research. Journal of Management Information Systems 24(3), 45-77 (2007)

Peleg, M., et al.: Comparing Computer-interpretable Guideline Models: A Case-study Approach. Journal of the American Medical Informatics Association 10(1), 52-68 (2003)

Pfeiffer, D., Becker, J.: Semantic business process analysis: building block-based construction of automatically analyzable business process models, Westfälische Wilhelms-Universität (2008)

Pfeiffer, D., Gehlert, A.: A framework for comparing conceptual models. In: Proceedings of the Workshop on Enterprise Modelling and Information Systems Architectures (EMISA 2005), pp. 108-122 (2005)

Rosemann, M.: Komplexitätsmanagement in Prozeßmodellen. Gabler, Wiesbaden (2002)

Rotter, T., et al.: A systematic review and meta-analysis of the effects of clinical pathways on length of stay, hospital costs and patient outcomes. BMC Health Services Research 8(1), $265(2008)$ 
Schlieter, H., Esswein, W.: From Clinical Practice Guideline to Clinical Pathway - Issues of Reference Model-Based Approach. In: Camarinha-Matos, L.M., Boucher, X., Afsarmanesh, H. (eds.) PRO-VE 2010. IFIP AICT, vol. 336, pp. 251-258. Springer, Heidelberg (2010)

Schnabel, M., et al.: Von der Leitlinie zum Behandlungspfad. Der Chirurg 74(12), 1156-1166 (2003)

Seyfang, A., Miksch, S.: Modelling Diagnosis and Treatment. International Journal of Clinical Monitoring and Computing 1 (2007)

Sinz, E.J.: Architektur von Informationssystemen. In: Rechenberg, P., Pomberger, G. (eds.) Informatik-Handbuch, pp. 1035-1046. Hanser, München (1999)

Wittgenstein, L.: Tractatus logico-philosophicus: philosophische Untersuchungen. ReclamVerlag, Leipzig (1922)

Wollersheim, H., Burgers, J., Grol, R.: Clinical guidelines to improve patient care. The Netherlands Journal of Medicine 63(6), 188-192 (2005)

Yin, R.K.: Applications of case study research. SAGE, London (2003)

Yu, E.: $\mathrm{i}^{*}$ an agent oriented modeling framework, Toronto (2011),

http: / / www. cs. toronto. edu/km/istar/ (download: 16.04.2011) 